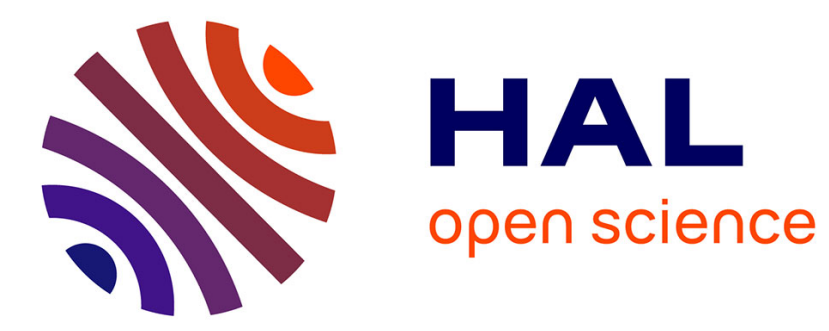

\title{
Cohesive stress heterogeneities and the transition from intrinsic ductility to brittleness \\ Döme Tanguy
}

\section{To cite this version:}

Döme Tanguy. Cohesive stress heterogeneities and the transition from intrinsic ductility to brittleness. Physical Review B: Condensed Matter and Materials Physics (1998-2015), 2017, 96, pp.174115. 10.1103/PhysRevB.96.174115 . hal-02295192

\section{HAL Id: hal-02295192 \\ https://hal.science/hal-02295192}

Submitted on 11 Dec 2019

HAL is a multi-disciplinary open access archive for the deposit and dissemination of scientific research documents, whether they are published or not. The documents may come from teaching and research institutions in France or abroad, or from public or private research centers.
L'archive ouverte pluridisciplinaire HAL, est destinée au dépôt et à la diffusion de documents scientifiques de niveau recherche, publiés ou non, émanant des établissements d'enseignement et de recherche français ou étrangers, des laboratoires publics ou privés. 


\title{
Cohesive stress heterogeneities and the transition from intrinsic ductility to brittleness
}

\author{
D. Tanguy $1, *$ \\ ${ }^{1}$ Univ Lyon, Université Claude Bernard Lyon 1, CNRS, \\ Institut Lumière Matière, F-69622, VILLEURBANNE, France
}

(Dated: November 7, 2017)

\begin{abstract}
The influence of nanoscale cavities on the fracture of the $\Sigma 33\{554\}[110]$ symmetrical tilt grain boundary is studied by atomistic simulations. The crack crystallography is chosen such that dislocation emission is easy. A transition from a ductile behavior of the tip to a brittle one is obtained for a dense (coverage beyond $15 \%$ and inter-cavity spacing smaller than $4 \mathrm{~nm}$ ) distribution of small cavities (sizes in between 1 and $2 \mathrm{~nm}$ ). The results are in good agreement with recent experiments from the literature. Even at the highest coverage, the character of the crack is highly sensitive to the initial position of the tip and a mixture of ductile and brittle responses is found. This complexity is beyond the usual criterion based on the drop of the work of separation with the amount of damage in the structure. It is shown that a heterogeneous cohesive zone model, with parameters extracted from the simulations and enriched with a criterion for plasticity, can explain the simulations and reproduce the transition. Additional simulations show that outside this range of small sizes and dense packing, which gives essentially a two dimensional response (either crack opening or infinite straigt dislocation emission), dislocation half loops appear for inter-cavity spacing starting at about $4 \mathrm{~nm}$. They constitute, together with regions of low covage/small cavities, efficient obstacles to brittle cracking. These results could be guidelines to designing interfaces more resistant to solute embrittlement, in general. The cohesive zone model is generic. Furthermore, the $\{554\}$ single crystal was used to determine to which extent the results depend on the details of the core structure vs. the cavity distribution. These elements show that the conclusions reached have a generic character.
\end{abstract}

\section{INTRODUCTION}

Fracture, in crystalline materials, is a competition between nanoscale debonding at the tip of flaws and the ease to release the stresses by plastic deformation. In metallic alloys, fracture is usually ductile, after large amounts of dislocations have been stored. Nevertheless, some impurities, such as hydrogen, helium or liquid metal, can cause a severe embrittlement. A model of such a transition from ductility to brittleness should address impurity effects on both cohesion and plasticity. The latter being multi-scale [1], a first approach has been to consider dislocation emission from a sharp crack tip, alone, in competition with its brittle extension [2, 3]. In this case, the mechanical load is represented by a scalar (k) which gives the intensity of the stress singularity at the tip. It scales like the applied stress times the square root of the crack length. Two critical values are defined: $\mathrm{k}_{I e}$ for dislocation emission, in traction (mode $\mathrm{I}$ ), and $\mathrm{k}_{I c}$ for cracking. $\mathrm{k}_{I c}$ is related to the energy release rate by $k_{I c}^{2} / E=W_{\text {sep }}$ where $\mathrm{E}$ is the Young's modulus and $\mathrm{W}_{\text {sep }}$ is the work of separation. For cleavage along a crystal plane (resp. an interface), $\mathrm{W}_{\text {sep }}$ is twice the surface energy (resp. twice the surface energy minus the interface energy). If $\mathrm{k}_{I e}$ is smaller than $\mathrm{k}_{I c}$, crack propagation is inhibited because dislocation emission blunts the tip before propagation initiates. The classical approach, to model the transition, is to determine a critical impurity concentration where $\mathrm{k}_{I c}$ crosses $\mathrm{k}_{I e}$. Some impurity effects on $\mathrm{k}_{I e}$ were evaluated by atomistic simulations. For

\footnotetext{
* dome.tanguy@univ-lyon1.fr
}

example, adsorbed $\mathrm{H}$ and $\mathrm{O}$ at crack tips in $\mathrm{Al}[\underline{4}$ ] tend to increase $\mathrm{k}_{\underline{I e}}$, with a significant influence of crack crystallography [5]. Local hydrides inhibit even more crack tip plasticity [6]. Substitutional solutes were also shown to modify significantly the energy barrier to emit half loops [7]. On the cohesion side, $a b$ initio calculations of ideal $\mathrm{W}_{\text {sep }}$, with intergranular impurities redistributed on surfaces, have been intensive. Of particular interest was understanding which of the elastic effect or "chemical effect" (essentially the difference in electronegativity between the host and the impurity) was responsible for the weakening or strengthening of the interfaces [8 12$]$. Larger embrittlements are obtained if the impurity chemical potential can be considered constant. In this case, the coverage increases during interface separation [1315]. Recently, more complex mechanisms are being considered where interstitial impurities are associated with vacancies. It has been proposed that these clusters [16 could aggregate or segregate to grain boundaries and induce damage [17, 18]. Their possible origin from dislocation reactions in the presence of hydrogen [19, 20], their slow diffusion [21] and their influence on dislocation mobility [22], have been investigated.

In this paper, we want to discuss their impact on intergranular cohesion, when they form nanoscale bubbles. The vacancy-hydrogen cluster being complex to handle with atomistic simulations, we drop the interstitial impurity and study the vacancies alone. First, the grain boundary and the various cavity configurations are presented. Second, the methods used for the fracture calculations, atomistic and continuous, are detailed. Then, $\mathrm{k}_{I e}$ and $\mathrm{W}_{\text {sep }}$ are calculated at increasing vacancy content until brittleness is reached. Finally, a continuous 
cohesive zone model is used to interpret and generalize the results. It shows that, beyond the variation of $\mathrm{k}_{I e}$ and $\mathrm{W}_{\text {sep }}$, it is the existence of cohesive stress heterogeneities which controls the brittle or ductile character of the crack. This paper deals essentially with small cavities (below $2 \mathrm{~nm}$ ) which trigger a two dimensional response of the system (either crack opening or infinite dislocation emission). A few simulations beyond this size were made and point at the inter-cavity size range where emission of half loops becomes important.

\section{CAVITY CONFIGURATIONS}

The grain boundary (GB) chosen is shown on Fig. 11. It has several interesting features. When the crack front is aligned with [110], an easy glide plane, containing the crack front, is available for emitting edge Shockley partial dislocations with a line parallel to the crack front. It provides good shielding and blunting of the tip. The glide plane is oriented at an angle of $65^{\circ}$ with respect to the GB plane, which is close to the angle giving the maximum resolved shear stress, according to elasticity $\left(70^{\circ}\right)$ [23]. Finally, it is a mixture of E structural units [24], and twin units (D structural units). The atoms in the twin units are almost coordinated like in the bulk and therefore more resistant to decohesion, while the core of the $\mathrm{E}$ unit is a site of preferential dislocation emission, in the absence of cracks [25]. This GB is therefore particularly resistant to fracture. Finding the conditions for its embrittlement would provide an upper bound for the critical damage.

According to the simulations in 24], the E structural units are present in symmetrical $\langle 110\rangle$ tilt grain boundaries with a misorientation angle in between $109.47^{\circ}$ and $180^{\circ}$, associated to $\mathrm{D}$ or $\mathrm{A}$ units, and with different degrees of distortions (7 examples are given in [24]). Other structural units compose the grain boundaries in the complementary misorientation angle range [26]. In addition to the simulations on the $\Sigma 33$ GB (Fig. 1), the $\{554\}$ single crystal is used to evaluate to which extent the fracture properties are related to the specificity of the E unit or to the cavities themselves.

The system is Aluminum, modeled by the potential given in [27]. The intergranular cavities are generated by off-lattice Metropolis Monte Carlo simulations (MC). The lowest energy structure of the grain boundary (GB) (Fig. 1a) is a reference lattice upon which space is decomposed in Voronoi cells [28]. Empty cells are vacancies. A micro-state is defined by a vector of site occupancies and a list of displacement vectors from the lattice site, when the cell is occupied. The MC simulations are a combination of first neighbor exchanges (the occupancies are switched and the displacement vector is conserved) between particles and vacancies and uniform sampling of the positions of the particles within their Voronoi cell. The temperature is fixed to $300 \mathrm{~K}$. The number of vacancies is constant during the MC run, but different runs

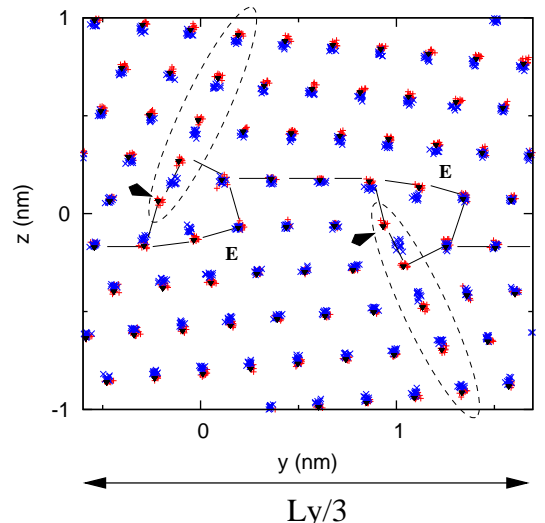

(a)

Figure 1. (Color online) (a) Unit cell of the $\Sigma 33\{554\}[110]$ symmetrical tilt grain boundary. The relaxation of the structure, at $300 \mathrm{~K}$, after the dissolution of two lines of vacancies is also represented (arrows). Ly is the size of the MC simulation box in y. (b) The cavities, projected in the crack plane $(\mathrm{x}, \mathrm{y})$, are shown together with the porosity profile for configurations (d) and (e). The cavities are numbered from 1 to 6 (top). Distances are in $\mathrm{nm}$.

Table I. Cavity sizes, in $a_{0}^{3}$, corresponding to the six GB configurations studied (labeled from a to e) in the absence of applied load. Configuration (s) is the $\{554\}$ single crystal with cavities from (e). The numbers, 1 to 6 , refer to the cavities, or peaks in the porosity profile in Fig. 2 The total number of vacancies (see text) is also given. The strain is zero.

\begin{tabular}{ccccccccc}
\hline \hline & 1 & 2 & 3 & 4 & 5 & 6 & total & Nvac \\
\hline $\mathrm{a}$ & 1.36 & 0.71 & 0.74 & 3.81 & 1.04 & 0.77 & 8.43 & 169 \\
$\mathrm{~b}$ & 0.74 & 1.33 & 0.33 & 1.01 & 0.85 & 1.14 & 5.40 & 206 \\
$\mathrm{c}$ & 1.38 & 2.10 & 0.81 & 0.83 & 4.42 & 2.00 & 11.56 & 204 \\
$\mathrm{~d}$ & 1.39 & 1.99 & 0.67 & 1.03 & 4.06 & 1.82 & 10.96 & 229 \\
$\mathrm{e}$ & 1.87 & 1.81 & 1.39 & 0.77 & 4.07 & 1.64 & 11.55 & 224 \\
$\mathrm{~s}$ & 1.88 & 1.74 & 4.97 & 1.16 & 5.55 & 2.22 & 17.52 & 257 \\
\hline \hline
\end{tabular}

have different numbers of vacancies.

The simulation box is composed of 3 unit cells in the $y$ direction $\langle\overline{2} 25\rangle\left(\mathrm{Ly}=\sqrt{33} \mathrm{a}_{0}\right), 12$ in the $\mathrm{x}$ direction $\langle 110\rangle$ $\left(\mathrm{Lx}=12 \sqrt{(2)} / 2 \mathrm{a}_{0}\right)$ and 5 in the $\mathrm{z}$ direction $\langle 5 \overline{5} 4\rangle$, for a total of 23256 atoms. The thermally exited zone in limited to 1 CSL (coincidence site lattice) cell on each side of the GB plane, for a total of 4644 atoms. Periodic boundary conditions are imposed in $\mathrm{x}$ and $\mathrm{y} .1 \%$ of the moves are trial exchanges. $99 \%$ are displacement moves, with a success rate of the order of $50 \%$ (the maximum amplitude for the displacements is $0.045 \mathrm{a}_{0}$ - the lattice parameterat $\mathrm{T}=300 \mathrm{~K})$. The volume of the system is constant. An initial strain is imposed by uniformly stretching the box perpendicular to the interface.

The simulation box contains 6 lines of E structural units in the GB plane (xy), oriented along the tilt axis ( $\mathrm{x}$ direction). The core of the $\mathrm{E}$ unit contains one site which 

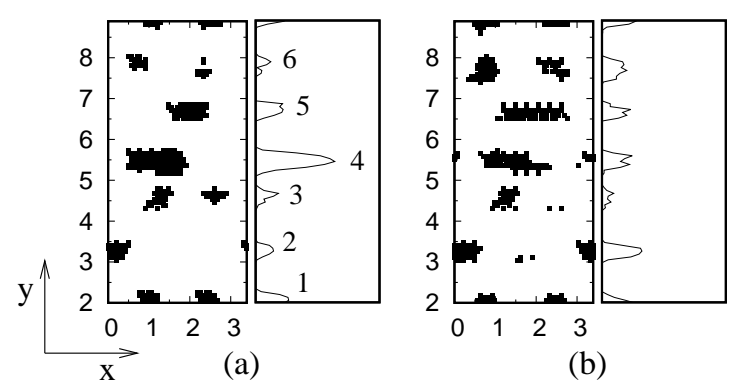

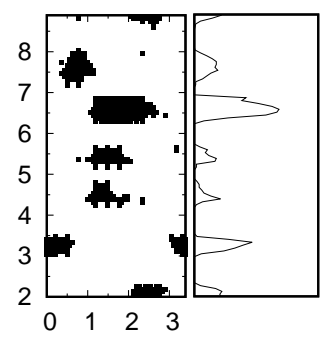

(c)

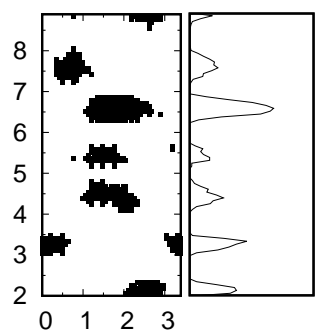

(e)

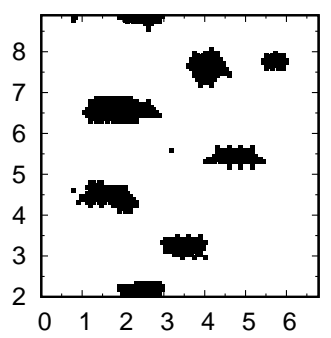

(f)

Figure 2. Cavity configurations (a), (b), (c) (e) and (f) (distances are in nm) obtained by Monte Carlo simulations. The "porosity profiles" are also given. The cavity labels (which corresponds to the E units or the former vacancy lines) are given on the profile of (a). The strain is zero.

absorbs vacancies preferentially (arrows on Fig. 1a), with a segregation energy $\Delta E_{\text {seg }}=-0.3 \mathrm{eV}$ at zero stress. The vacancy-vacancy interaction, along the tilt axis, is attractive with a pair energy of $-0.1 \mathrm{eV}$, at zero stress. Therefore, when the vacancies meet the GB, they are trapped and agglomerate to form linear chains (Fig. 3b), oriented along the tilt axis. When the strain is large enough (beyond 4\%), these lines are destabilized and vacancy clusters form. Each E structure line contains, at least, one cluster, slightly off the GB plane (alternatively above and below it). The cavities, and their volume, are determined by using a fine cubic grid. Each time the center of a cell is within the atomic radius of a particle, the cell is considered occupied. The cell size is adjusted such that no voids are detected in a perfect fcc crystal, i.e. octahedral and tetrahedral sites do not appear as voids. Then, in the case of the GB vacancy aggregates, the cavities are simply clusters of empty cells. The grid size is $1 / 4$ of the first neighbor distance. The six configurations selected for the fracture calculations are shown on Fig. 10 and 2] A "porosity profile" is also associated to each picture. It gives the number of empty cells per line. The integral over each peak gives the cavity volume reported in Tab. I. The cavities are labeled from 1 to 6 (Fig. 2a). Configuration (d) derives from (c), with more vacancies inserted during the MC process. Even if the cavity size distribution is similar, each cavity shape is different. Configuration (e) derives from (d), with a marked increased in the size of cavity 3 , which is shown to play a crucial role in embrittlement below. (f) derives from (e) by multiplying the $\mathrm{x}$ dimension of the system by a factor 2 , and shifting one cavity out of two in the $\mathrm{x}$ direction. A chessboard structure is formed, at half density, keeping the same cavities as (e), which enables studying the effect to inter-cavity spacing on dislocation emission. The $\{554\}$ single crystal is studied without cavities and with the cavities of configuration (e). The single crystal with cavities is constructed in the orientation of the upper part of the system with the GB. The vacancies of the lower part are symmetrized with respect to the GB plane, translated back to their original position and then inserted in the single crystal. Those of the upper part are simply inserted.

Note that the relaxations in the GB are large: (i) no cavities are detected when the vacancies are in the form of lines; (ii) the total cavity volume (Tab. I), when transformed in a number of vacancies by dividing by the volume of an atom in the fcc structure $\left(0.25 a_{0}^{3}\right)$, is much smaller than the number of vacancies detected by the reference lattice (column Nvac in Tab. II). It means that the density is much smaller than what the volume of the cavities alone suggests. This might be at the origin of the large volume expansion of the cavities during mechanical loading. They grow elastically almost up to $5 \mathrm{GPa}$ (the inspection of the structure did not show any defect creation). The volume changes are linear and can be as high as a factor two.

One can wonder what is the driving force for the formation of the bubbles. Indeed, in the bulk, the vacancyvacancy pair interaction is slightly repulsive [29, 30] and experiments suggest that bubbles can be stable, in the presence of hydrogen, only is the case of severe vacancy supersaturation [31]. The formation of clusters, in the bulk, was studied recently 32] at the atomic scale. The authors have calculated activation energies and binding energies obtained with the same potential as the one used here [27]. The agreement with DFT is good, at least up to clusters composed of 5 vacancies. Kinetic Monte Carlo simulations, on a rigid lattice, based on such energetic parameters show that large clusters can form at high initial vacancy concentrations and intermediate temperatures, even if the divacancy is not stable. To understand the driving force for the formation of the bubbles in the GB, starting from linear clusters, we evaluate the energy difference $(\Delta \mathrm{E})$ between two configurations having the same number of vacancies (Fig. 3): a bubble, of different size, extracted from the MC simulations and a linear cluster composed of the same number of vacancies, placed in the most favorable position in the $\mathrm{E}$ unit. The latter is the lowest energy structure at zero stress. First, without strain, the bubbles are unstable, whatever their size $(\Delta \mathrm{E}$ is positive on Fig. 3a). The energy difference roughly goes up with the number of vacancies (nVac). The energy difference, per vacancy, fluctuates around a value similar 
to the opposite of the segregation energy $(0.3 \mathrm{eV})$. We can therefore interpret this energy difference by imagining the following path to transform the linear chain into a bubble: a vacancy is taken from the line and is put back on a bulk site, with an energy increase of $0.3 \mathrm{eV}$ (minus the segregation energy). Then this vacancy is aggregated into a already existing small bubble. According to 32] the associated energy variation is marginal. Therefore, up to that point, our findings for the GB are coherent with the bulk behavior. Second, an applied strain perpendicular to the GB tends to decrease this energy difference (Fig. 3 3 ). To analyze this effect, we consider the energy difference between the system containing the vacancies (either in the form of a bubble or in the form of chains) and a reference, at the same strain, without vacancies. We call this energy difference: the excess energy. The EAM potential is such that the potential energy is split in between the different atoms of the system. So the excess energy per site can be used. It shows how a specific spatial vacancy arrangement relaxes the energy stored in the system. This excess energy is split in two: the sum on the atoms in first neighbor position of the vacancies, which represents the core effect of the vacancies, and the rest which represents the elastic response. This decomposition, done for two cluster sizes (clusters $1_{a}$ and 4 on Fig. 3 a), shows without ambiguity that the $\Delta \mathrm{E}$ energy decrease comes from the elastic energy and not the core energy. Furthermore, it is the configuration where the vacancies are arranged in the form of a line which generates an excess elastic energy. In the bubble case, the elastic energy is similar to the one without vacancies. The excess energy maps (Fig. 3r) show that the energy is localized along the planes where the vacancies are delocalized (see also the dashed lines on Fig. 19).

\section{FRACTURE METHODOLOGY}

The Cleri set up 33, 34 is used for the calculation of the stress intensity factor for dislocation emission $k_{I e}$. It consists in a "large", square, box containing an internal crack (Fig. 4a). A stable crack is created by removing atoms, typically two layers. Therefore, the tip has a rectangular shape (Fig. 4b), initially. The cavities are introduced in the system by replicating the MC box 8 times (24 structural units) in the y direction, for a total length of $137.8 \mathrm{a}_{0}(55 \mathrm{~nm})$. The MC configurations are quenched and no further MC moves are done during fracture. The dimension in the $\mathrm{z}$ direction is $154.17 \mathrm{a}_{0}(61.7 \mathrm{~nm})$. Two rigid grips of thickness $4.06 \mathrm{a}_{0}(1.62 \mathrm{~nm})$ are used at the top and bottom of the box. The dimension in the $\mathrm{x}$ direction (the thickness) is the same as the MC box: $12 * 1 / 2$ $\langle 110\rangle$ lattice vectors, i.e. $8.48 \mathrm{a}_{0}(3.39 \mathrm{~nm})$. The initial half crack length (a) is $16.3 \mathrm{a}_{0}(6.5 \mathrm{~nm})$. Various positions of the crack tip are chosen to sample the response at different locations along the GB, with or without cavities. Periodic boundary conditions are applied in $\mathrm{x}$ and y. The total number of atom is 719616. This "small"
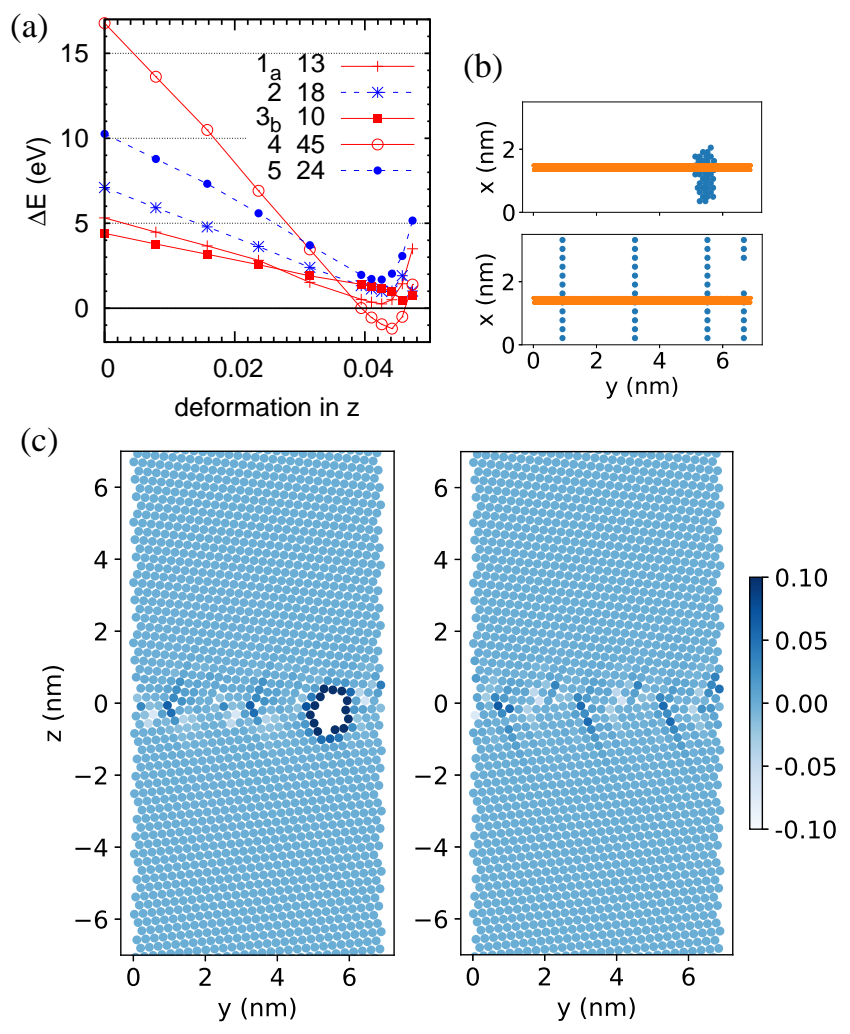

Figure 3. (Color online) Stability of cavities: (a) Energy difference between a system containing a bubble and a system containing the same number of vacancies aligned along the tilt axis, as a function of the strain perpendicular to the interface. The cavity labels and the number of vacancies appear in the curves' labels. They are taken from configuration a. (b) A view perpendicular to the interface showing the vacancies clustered (cavity 4) and the same number of vacancies aligned along the tilt axis in different $\mathrm{E}$ units. The transversal cuts shown in (c) are represented by horizontal lines. (c) Excess energy map (difference of site energy between a configuration containing vacancies and a reference without vacancy at the same strain of $3.2 \%$, in $\mathrm{eV}$ ) for the two systems shown on (b).

system is used for calculating $k_{I e}$ values. The energy is minimized after each increment of displacement on the rigid border, in the $\mathrm{z}$ direction. The crack is loaded in mode I. The stress is calculated on every atom from the Virial formula. The stress intensity factor $(\mathrm{k})$ is obtained by fitting the shear stress field to the analytical solution for a internal crack in an infinite medium (35] p. 6), in a window close to the tip. It is necessary to use the shear component because the Virial stress does not give atomic stresses which compares well with continuum in the GB plane or at the surface of the cavities. The shear stress takes large values at an angle from the GB plane which avoids such problems. Once the fit is done, $\mathrm{k}$ is extracted from the elastic solution. The system size effect was tested by doubling the system size and by doubling the system size and the crack size (half crack size 13 $\mathrm{nm})$. The corresponding $k_{I e}$ values for the configuration 
without cavities are shown on Fig. [5] and [36]. Three system sizes were also used for configurations (c) and (e) (Fig. 5). With this set up, the $\mathrm{k}$ dominated stress field expands about $18 \mathrm{a}_{0}(7 \mathrm{~nm})$ from the crack tip (Fig.5 in [37]), significantly larger than the process zone when dislocations are emitted from the crack tip. As a consequence, the low $k_{I e}$ values are connected to the elastic energy release rate in the usual way. Indeed, we have always had good agreement between such $\mathrm{k}$ values and the Griffith theory [34], or the Rice theory [38]. High $k_{I e}$ values correspond to configurations where the process zone has very significantly expanded (up to a value in between 4 and $6 \mathrm{~nm}$ ) and becomes comparable with the "small" crack length of the simulations. In this case, it can be shown [36] that the small cracks $(a=6.5 \mathrm{~nm})$ are not in the "k-controlled" domain, while the "large" cracks $(\mathrm{a}=13 \mathrm{~nm})$ are just at the beginning of the domain. As a consequence, the $k_{I c}$ values, extracted from the work of separation $W_{\text {sep }}$ with $k^{2} / E=W_{\text {sep }}$, can only be compared rigorously to the largest $k_{I e}$ values (which characterize the initiation of the fracture process zone) in the "large crack" case (Fig. 10). The single crystal was tested only the "long" crack configuration.

The crystallography corresponds to a slightly rotated \{111\} single crystal (dotted lines on Fig. 4a). The easy glide direction $\langle 1 \overline{1} 2\rangle$, in the $(\overline{1} 11)$ plane, receives a high resolved shear stress (Fig. 4a). Straight edge partial dislocations can be emitted directly from the tip, on the right side of the crack, along this slip system (Fig. 40 and c). This is the intrinsically ductile crack tip where the competition between dislocation emission and crack propagation is studied. The other crack tip is brittle 39, 40], but propagation is only observed when a high load is applied, with the left tip constrained (see below), except for configuration (e) (see movie in [36]).

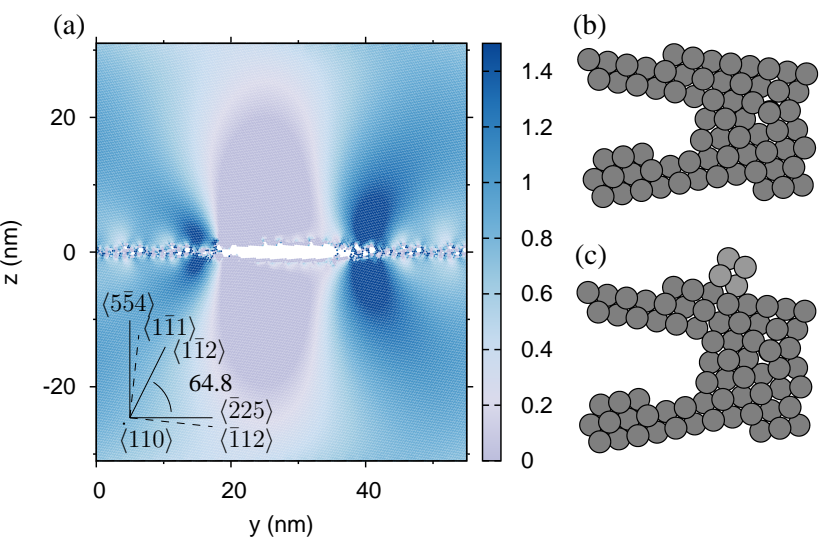

Figure 4. (Color online) (a) The map gives the resolved shear stress $(\mathrm{GPa})$ in the easy glide direction $\langle 1 \overline{1} 2\rangle$ in the plane (111). A crack tip is shown before (b) and after emission of a dislocation (c), from the "corner" of the tip.

The "thin strip" set up [41] was used for calculating the work of separation $\left(W_{\text {sep }}\right)$. The system is wide and narrow. The minimal box that was used contains 92 CSL in the $\mathrm{y}$ direction, 16 in the traction direction $(\mathrm{z})$ and the same thickness as above. The dimensions are 528.5 $\mathrm{a}_{0}(211 \mathrm{~nm}) \times 65 \mathrm{a}_{0}(26 \mathrm{~nm})$. The initial crack length is $132 \mathrm{a}_{0}(53 \mathrm{~nm})$. The number of atoms is 1162512 . The system size was doubled for configuration (e) to check that the size effect on $\left(W_{\text {sep }}\right)$ was acceptable.

Finally, continuum mechanics is used for analyzing the results of the atomistic simulations. The fracture mechanisms ahead of the crack tip are captured by a relation $\left(\sigma_{C Z}(\delta, y)\right)$, which connects the normal stress along the interface and the non elastic opening $(\delta)$. The opening profile $(\delta(y))$ is twice the displacement $u_{z}(y)$. It is connected to $B(y)$, the infinitesimal dislocation distribution of the crack fracture process zone, by:

$$
\delta(y)=\int_{y}^{\infty} B(x) d x=2 u_{z}(y)
$$

Continuum mechanics gives the traction stress profile along the crack plane $\sigma(y)$ which is generated by $B(y)$ [35]:

$$
\sigma(y)=\frac{\mu}{2 \pi(1-\nu)} \int_{-\infty}^{\infty} \frac{B\left(y^{\prime}\right)}{y-y^{\prime}} d y^{\prime}
$$

At equilibrium, within the fracture process zone, the equality $\sigma(y)+\sigma_{a p p}=\sigma_{C Z}(\delta(y), y)$, where $\sigma_{a p p}$ is the applied stress, is verified. With a parametrized $\sigma_{C Z}(\delta, y)$, it constitutes a robust mesoscale model of the fracture process zone which gives the crack opening profile, as a function of the applied load. The interest is in finding the $\sigma_{C Z}$ parametrization which reproduces the atomistic results as it provides, at the same time, a model applicable in continuum calculations at higher scales, and an understanding of the essential features which rule the fracture behavior. Details about the parametrization and the numerical resolution are now given.

As a first step, equations 1 and 2 were used to transform the atomistic crack opening profiles, into a stress profile along the interface. Combined to $\delta(y)$, gives the shape of the $\sigma_{C Z}(\delta(y), y)$ relation. The data is noisy, but, at low loads, when the process zone is small, $\sigma_{C Z}(\delta)$ seems to follow the universal binding law of Rose [42] (for a precise determination of cohesive zone relations from atomistics see [43]). At higher loads, $\sigma_{C Z}(\delta)$ seems linear. Therefore, for simplicity, we chose a linear model, with two parameters: the maximum cohesive stress $\left(\sigma_{\max }\right)$ and the critical opening $\left(\delta_{c}\right)$, where the stress becomes zero. The originality is that $\sigma_{\max }$ is a function of the position along the GB. $\sigma_{\max }(y)$ is taken constant by segments. The average value of $\sigma_{\max }(y)\left(\overline{\sigma_{\max }}\right)$ was obtained by uniaxial traction simulations, without crack, on small systems containing the full cavity population (Tab. III), such as the ones shown on Fig. 2. The values of $\delta_{c}$ (Tab. III) are extracted from the work of separation obtained by atomistic simulations (Tab. III) with the relation $W_{s e p}=1 / 2 \times \overline{\sigma_{\max }} \times \delta_{c}$. The $\sigma_{\max }(y)$ profile, of the heterogeneous cohesive zone model, is given in the result section. 
Having set the cohesive zone model, continuum mechanics is used to obtain the equilibrium of an internal crack of length $2 \mathrm{a}$, with a nonlinear process zone ahead of the tip, submitted to an external load $\sigma_{a p p}$, at infinity. The elastic medium is isotropic with the parameters $\mu$ and $\nu$ for aluminum. The dislocation distribution is given by the Muskhelishvili equation [35] (p. 106):

$$
B(y)=-\frac{2(1-\nu) \sqrt{c^{2}-y^{2}}}{\pi \mu} \int_{-c}^{c} \frac{\sigma\left(y^{\prime}\right) d y^{\prime}}{\left(y-y^{\prime}\right) \sqrt{c^{2}-y^{\prime 2}}}
$$

and the parameter $\mathrm{c}$ which gives the length of the process zone (PZ) $L_{P Z}=c-a$ is taken from:

$$
\int_{-c}^{c} \frac{\sigma(y) d y}{\sqrt{c^{2}-y^{2}}}=0
$$

with: $\mathrm{B}(\mathrm{y})=0$ for $|y|>c, \sigma(y)=-\sigma_{a p p}$ for $|y|<a$ and $\sigma(y)=\sigma_{Z C}(\delta(y), y)-\sigma_{a p p}$ for $a<|y|<c . \sigma_{Z C}(\delta(y), y)$ is the a linear function of $\delta$, with the parameter $\sigma_{\max }$ depending on $\mathrm{y}: \sigma_{Z C}(\delta, y)=\sigma_{\max }(y)\left(\delta / a_{c}-1\right) . \mu$ is the shear modulus of the Al potential $(30.68 \mathrm{GPa})$ and $\nu$ the Poisson ratio (0.332).

Technically, the integral in Eq. 3 is calculated numerically by the Gauss-Chebyshev quadrature [44] (Eq. 7.55 p. 380). Equations 1 and 3 are solved iteratively $(\delta(y)$ from Eq. 11 is inserted in $\sigma_{C Z}$ in Eq. 3, with a first step where $\sigma(y)=\overline{\sigma_{\max }}$ ) for a range of c values. The solution is the one which satisfies Eq. 4.

\section{INFLUENCE OF THE CAVITIES ON $k_{I e}$}

The critical stress intensity factor $\mathrm{k}_{I e}$ is very dependent on the location of the tip in the GB and on the various cavity configurations (Fig. 5). In the absence of cavities ("no vac" on Fig. [5 and [36]), a first group of values correspond to a dislocation emission from an $\mathrm{E}$ structural unit (Fig. 19) at one atomic distance from the tip, at $k_{I e} \sim 0.35 \mathrm{MPa} \mathrm{m}^{1 / 2}$. The other configurations lead to an easier emission from the corner of the tip at $k_{I e} \sim 0.27 \mathrm{MPa} \mathrm{m}{ }^{1 / 2}$ (Fig. 45). For comparison, emission from the tip in the $\{554\}$ single crystal case, i.e. in the absence of E structural unit, occurs at $0.31 \mathrm{MPa}$ $\mathrm{m}^{1 / 2}$ (triangle in Fig. 5). Before emission, the GB region is elastic. Introducing cavities apparently increases $k_{I e}$ (Fig. 5), with a large sensitivity to the position of the tip. Two clearly distinct mechanisms operate: (i) emission from the corner of the tip, similar to the nondefective GB, or (ii) the growth of the cavities and the gradual formation of a fracture process zone (PZ). A similar behavior, within a similar k range, is obtained for the single crystal with cavities inherited from configuration (e) (triangles in Fig. 5).

These mechanisms are precisely monitored in the simulations by following the shear localization. For this, we introduce the displacement shift profile $\Delta \tilde{u}_{z}^{i}(y)$, which is also a key ingredient for providing a plasticity criterion to the cohesive zone model. Consider the displacement in the traction direction of two consecutive atomic rows in the crack plane, $u_{z}^{i}$ and $u_{z}^{i+1}$, projected in the easy glide direction ( $\alpha$ is the angle between the $\mathrm{z}$ direction and the glide direction in Fig. 6a). The difference $\Delta \tilde{u}_{z}^{i}$, neglecting the contribution of the displacements in $\mathrm{x}$ and $\mathrm{y}$, is a measure of the relative displacement of the atoms above and below a glide plane that would intercept the crack plane in between rows $i$ and $i+1$. The typical variation of the energy with $\Delta \tilde{u}_{z}^{i}$ is schematically represented on Fig. 6b [45]. Its derivative is the resistance shear stress produced by the lattice. It reaches a maximum value for approximately $1 / 2$ of the value corresponding to the maximum of the energy, i.e. 1/4 of the displacement shift created by the fully formed dislocation: $1 / 6\|\langle 112\rangle\| a_{0} / 4 \sim 0.041 \mathrm{~nm}$. When the applied load reaches $\mathrm{k}_{I e}$, the incipient dislocation has expended to the point where the maximum resistance is exceeded, the dislocation pops out and $\Delta \tilde{u}_{z}^{i}$ abruptly jumps above $0.041 \mathrm{~nm}$ at the emission point (see appendix). This value is therefore a threshold for dislocation emission. Two representative examples of emission at a low and high $k_{I e}$ are given: Fig. 6r corresponds to the emission from the tip and Fig. G $\mathrm{d}$ corresponds to emission ahead of the tip after the PZ has expended by the growth of the cavities. In this case, a peak appeared first at the tip and then in front of cavity 6 and 1, without overcoming the threshold until a fully formed dislocation is emitted in front of cavity $3\left(\Delta \tilde{u}_{z}^{i} \sim 0.08 \mathrm{~nm}\right)$. It was found, for configurations (c) and (d), that the neighborhood of cavity 3 is a very cohesive region which always leads to dislocation emission whatever the initial position of the tip.

With the Rice and Thomson model [46] in mind (the crossing of $k_{I e}$ and $k_{I c}$ mentioned in the introduction), we expected a continuous change in $k_{I e}$ with the increasing amount of damage in the GB. The outcomes of the simulations are more complex. First, the heterogeneity of the GB structure itself gives a range of $k_{I e}$, depending on the initial position of the tip, even in the absence of cavities. Second, with increasing damage, the trend is a spreading of this range towards higher values. In every configuration tested, it is possible to find a crack tip position where dislocation emission is easy. The high values of $k_{I e}$ are always associated to the onset of cavity growth, even if it does not lead to fracture. The pinning of the dislocations by the cavity [47] is weak in comparison to the stress concentration at the crack tip and is not responsible for the increase of $k_{I e}$. Brittle propagation is only obtained in configuration (e) (4 crack tip positions lead to propagation, out of 10 , with the long crack system). The "same" cavity configuration introduced in the $\{554\}$ single crystal produces a range of $k_{I e}$ values similar to the GB case. Nevertheless, brittle crack propagation is never obtained. 


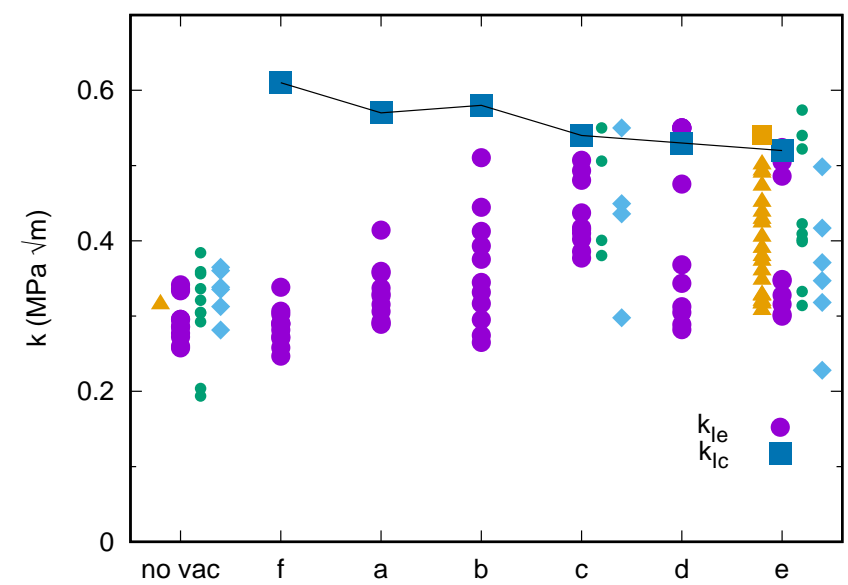

Figure 5. (Color online) Critical stress intensity factor for emission of a Shockley partial $\left(k_{I e}\right)$ for the different vacancy configurations, ordered by increasing number of vacancies per surface area. For each configuration several crack tip positions are tested. The different system sizes are: small system-small crack $(\bigcirc)$ (half crack length $\mathrm{a}=6.5 \mathrm{~nm}$ ), large system-small crack (०), large system-large crack GB $(\diamond)(\mathrm{a}=13 \mathrm{~nm}),\{554\}$ single crystal $(\triangle)$. The positions of the tip are given in the appendix. The stress intensity factor for cracking $\left(k_{I c}\right)$ is given for the GB ( $\square$ ) and for the single crystal.

\section{INFLUENCE OF THE CAVITIES ON THE WORK OF SEPARATION}

Cohesion was characterized by measuring the work of separation by crack propagation simulations in the "thin strip" geometry [41] (section [III). Still with the Rice and Thomson model in mind, we search for a trend giving the decrease of the work of separation $\left(W_{\text {sep }}\right)$ with the amount of damage in the grain boundary. As a first approach, which is refined in the final discussion, the damage is characterized by the total number of vacancies in the GB (Tab. I). Damage increases from configuration (a) to (e). It was shown in the previous section that configurations (a) to (d) are intrinsically ductile, even if some cavity growth can be obtained. In principle, if dislocations are emitted, it is not possible to evaluate $W_{\text {sep }}$ and therefore the decrease in $k_{I c}$ cannot be quantified. We have developed specific equations of motions for this case: dislocation emission is constrained, within Molecular Statics, by incorporating configurational forces which compensate the resolved shear stress in the direction of easy glide [34]. Constrained atoms are not directly within the FPZ, where they could modify the inter-cavity fracture mechanisms, but on the side (in a strip starting at a distance $2.7 \mathrm{a}_{0}$ from the interface plane and ending at $3.3 \mathrm{a}_{0} / 2.7-5.1 \mathrm{a}_{0}$ for (f) and the single crystal). Events involved in fracture, other than Shockley partial emission in the direction of maximum resolved shear stress, are free to occur. A list of such mechanisms is given below. The values of $W_{\text {sep }}$, obtained with this method, are given in Table II First, the values are two to three times (a)
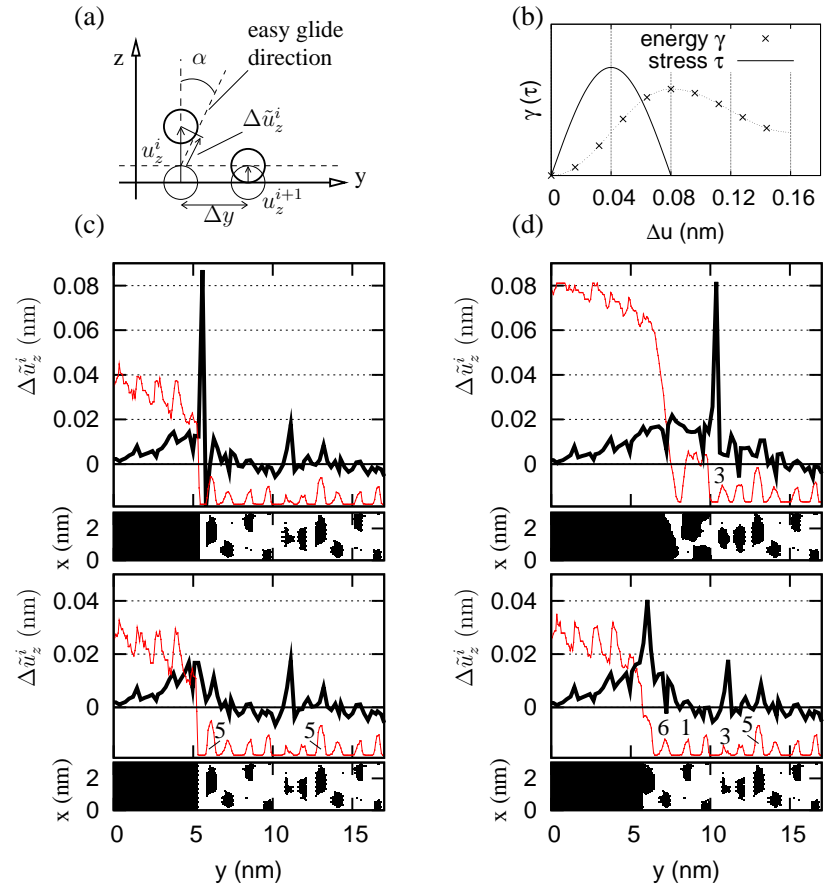

(d) $\Delta \mathrm{u}(\mathrm{nm})$

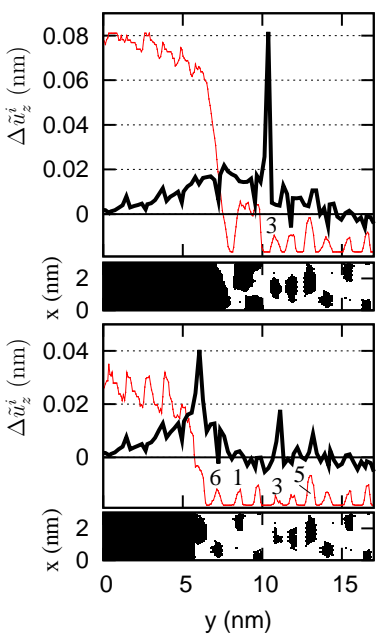

Figure 6. (Color online) The displacement shift, $\Delta \tilde{u}_{z}^{i}(\mathrm{~nm})$, is defined on (a). (b) Schematic representation of the energy profile $(\gamma)$ for a displacement shift $\Delta u$ along $\langle 112\rangle\{111\}$ and the opposing shear stress $(\tau)$. The evolution of the $\Delta \tilde{u}_{z}^{i}$ profile is shown on (c) and (d) for two different crack tips, labeled (3) and (1) on Fig. 5]. The cavity configuration is (d). Load increases from bottom to the top. The porosity profile is superimposed. The corresponding views of the cavities, in the crack plane (xy), are also added to illustrate their growth and coalescence. Cavity labels are specified when necessary.

higher than the ones corresponding to a rigid separation. Such large differences have already been reported in the past [48] and interpreted as a "lattice" or "bond trapping", meaning that the crack path does not necessarily leads to flat surfaces with a low energy. In our case, voids grow and coalesce with the crack (Fig. 6d), involving self interstitial injection, but also local glide in between the cavities. The energetics is completely different from the cleavage case [42]. The $W_{\text {sep }}$ values are coherent with [40] which finds $6 \mathrm{~J} / \mathrm{m}^{2}$ for a brittle orientation (without initial cavities). Second, there is a gradual decrease of $W_{\text {sep }}$ with the total void content, until true brittle propagation is obtained for configuration (e). Note that the value of $W_{\text {sep }}$ for (e) is obtained without constraint and is well inline with the others. Furthermore, the fracture mechanism is the same as in the simulations with constraints, which indicates that a physical fracture mechanism was obtained with this method.

The simulations show that the transition is not given by the comparison of $\mathrm{k}_{I c}$ with $\mathrm{k}_{I e}$. Indeed, even if $\mathrm{k}_{I c}$ decreases with the total number of vacancies, it is still in the upper range of the $\mathrm{k}_{I e}$ values (Fig. (5). Furthermore, 
the response is very dependent on the initial position of the tip. The case of configuration (e) is the most striking. A sampling of 20 different initial crack tips (Fig. 10) was done. In the case of the large crack, in the large system (Fig. 10 ), 7 crack tips out of 10 lead to the initiation of a brittle crack process zone and 3 lead to the immediate emission of a dislocation, right at the crack tip. 4 out of 7 brittle PZ lead to a true brittle propagation (crack runs for a long distance [36]).

Before we conclude about the cavity size and density necessary for embrittlement, a rationalization of this mixture of brittle and ductile events is necessary. To achieve this, a cohesive zone model is used to analyze the results of the simulations. A sound quantification of the embrittlement will be given in the final discussion.

Table II. The table gives the work of separation $W_{\text {sep }}\left(\mathrm{J} / \mathrm{m}^{2}\right)$ with the precision $\delta W_{\text {sep }}$, the average cohesive stress $\overline{\sigma_{\max }}$ $(\mathrm{GPa})$ and the corresponding critical opening $\delta_{c}(\mathrm{~nm})$ for a linear cohesive law. $k_{I c}$ is the Griffith stress intensity factor corresponding to $W_{s e p}$, for comparison with the $k_{I e}$ values of Fig. 5

\begin{tabular}{cccccc}
\hline \hline configuration & $\begin{array}{c}W_{\text {sep }} \\
\left(\mathrm{J} / \mathrm{m}^{2}\right)\end{array}$ & $\begin{array}{c}\delta W_{\text {sep }} \\
\left(\mathrm{J} / \mathrm{m}^{2}\right)\end{array}$ & $\begin{array}{c}\overline{\sigma_{\max }} \\
(\mathrm{GPa})\end{array}$ & $\begin{array}{c}\delta_{c} \\
(\mathrm{~nm})\end{array}$ & $\begin{array}{c}k_{I c} \\
(M P a \sqrt{m})\end{array}$ \\
\hline 111 & 1.52 & & 13.1 & 0.23 & 0.35 \\
$111 \mathrm{DFT}^{[14]}$ & 1.84 & & 12 & 0.31 & 0.39 \\
GB no vac rigid & 1.64 & & 11.2 & 0.29 & 0.37 \\
conf a rigid & 1.51 & & 10.7 & 0.28 & 0.35 \\
conf d rigid & 1.45 & & 10.3 & 0.27 & 0.34 \\
conf a & 4.00 & 0.03 & - & - & 0.57 \\
conf b & 4.06 & 0.03 & 6.31 & 1.28 & 0.58 \\
conf c & 3.56 & 0.02 & 6.15 & 1.16 & 0.54 \\
conf d & 3.46 & 0.02 & 6.10 & 1.13 & 0.53 \\
conf e & 3.27 & 0.05 & 6.00 & 1.09 & 0.52 \\
conf f & 4.54 & 0.05 & 7.10 & 1.28 & 0.61 \\
$554 \mathrm{e}$ & 3.59 & 0.05 & 6.77 & 1.06 & 0.54 \\
\hline \hline
\end{tabular}

\section{COHESIVE ZONE MODELING}

Finally, it is shown below that the complex response of the crack tip is reproduced and clarified with a simple, heterogeneous, cohesive zone model (CZ). It is proposed as an alternative to the comparison between $k_{I c}$ and $k_{I e}$. The technical aspects are given in section III. The model relates the non elastic traction stress along the interface $\left(\sigma_{C Z}(y)\right)$ to the crack opening $(\delta(y))$ by a linear relation $\sigma_{C Z}(y)=\sigma_{\max }(y)\left(1-\delta(y) / \delta_{c}\right)$. The originality is that the cohesive stress $\sigma_{\max }$ is made dependent on the position. Continuum mechanics is used to determine the equilibrium of an internal crack, under tension, with the nonlinear process zone described by $\sigma_{C Z}(y)$ ahead of the tip. In particular, it gives $\delta(y)$ as a function of the applied load. The model is further extended by incorpo- (a)

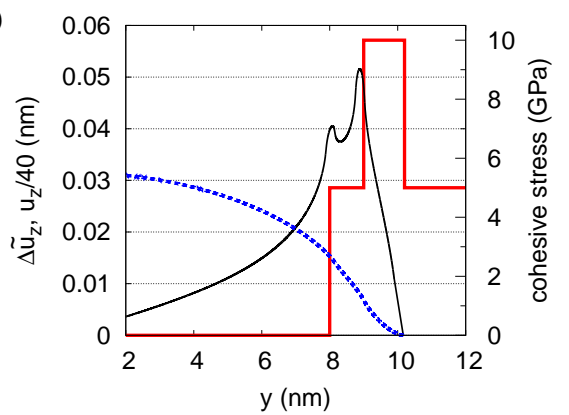

(b)

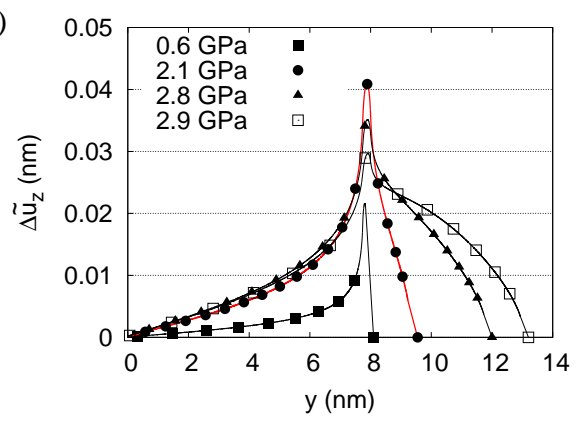

Figure 7. (Color online) (a) Crack opening $\left(\mathrm{u}_{z}\right)$ and displacement shift $\left(\Delta \tilde{u}_{z}\right)$ profiles corresponding to a cohesive zone model with one heterogeneity, shown on the cohesive stress profile. The crack tip is at $8 \mathrm{~nm}$. The process zone size is about $2 \mathrm{~nm}$. (b) Evolution of the $\Delta \tilde{u}_{z}$ profile with increasing applied load, showing the broadening of the PZ and the variation of the maximum value of $\Delta \tilde{u}_{z}$ : first an increase and then, beyond 2.1 GPa, a decrease.

rating a threshold on $\Delta \tilde{u}_{z}^{i}$, set to $0.04 \mathrm{~nm}$ according to atomistics, as a criterion for the onset of plasticity. For this, a continuous version of $\Delta \tilde{u}_{z}^{i}$ is used. It is the finite difference $\Delta \tilde{u}_{z}=\cos \alpha\left(u_{z}(y)-u_{z}(y+\Delta y)\right)$, where $\Delta y$ is the distance between two atomic rows on the crack surface, $u_{z}(y)=\delta(y) / 2$ and $\alpha$ is the angle between $\mathrm{z}$ and the glide plane (Fig. [6a). Anytime $\sigma_{C Z}(y)$ has a discontinuity, $\Delta \tilde{u}_{z}(y)$ has a local maximum. Figure $7 \mathrm{~b}$ shows an example with two discontinuities: first at the crack tip where the cohesive stress jumps to an non-zero value and second at the level of an heterogeneity ahead of the crack tip. Every peak in the $\Delta \tilde{u}_{z}(y)$ profile corresponds to a localization of shear deformation i.e. to an incipient dislocation.

When the applied load increases, the $\Delta \tilde{u}_{z}$ peak, at the tip, first increases, reaches a maximum and then decreases (Fig. 7b). In the mean time, the PZ size increases. To avoid dislocation emission at the tip, the $\Delta \tilde{u}_{z}$ peak should remain below the threshold for plasticity during the PZ evolution. For our model with a $\delta_{c}$ of $1.09 \mathrm{~nm}$, typical of the damage by nanoscale cavities (Tab. III), the calculations show that $\sigma_{\max }$ should drop below $5.5 \mathrm{GPa}$ in order to keep $\Delta \tilde{u}_{z}$ below $0.04 \mathrm{~nm}$. This is the criterion for the initiation of a brittle PZ. In particular, none of the uniform CZ models with parameters in Tab. II can initiate the formation of a brittle PZ. This is in contra- 

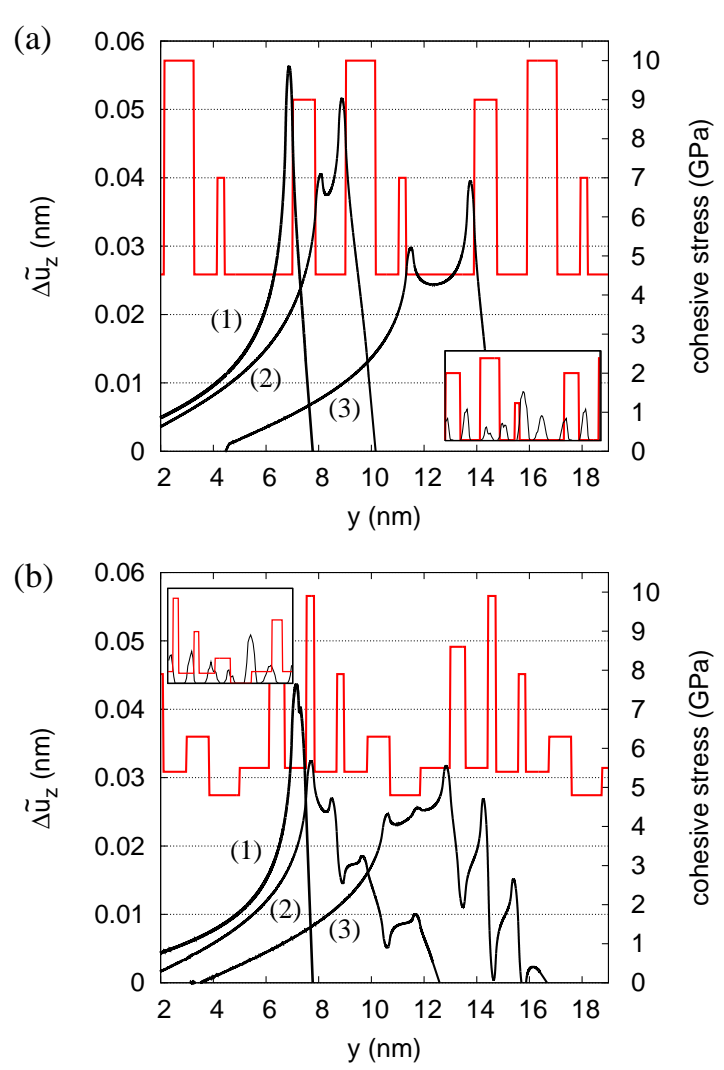

Figure 8. (Color online) Characteristic $\Delta \tilde{u}_{z}$ profiles for the cavity configurations (d) (Fig. a) and (e) (Fig. b) and three different tip positions. The crack tip location is always given by the position of the first peak on the $\Delta \tilde{u}_{z}$ profile. The cohesive stress profile $\left(\sigma_{\max }(y)\right)$ is superimposed (right axis in $\mathrm{GPa}$ ). The inserts show the correspondence between the porosity profiles and the $\sigma_{\max }$ profiles.

diction with the atomistics simulations from which the parameters were extracted. We show below that a heterogeneous $\mathrm{CZ}$ can reach a good agreement.

A parametric study [36] determined the characteristics of a $\sigma_{\max }$ heterogeneity, ahead of the crack tip, that could stop the brittle expansion of the PZ. The $\Delta \tilde{u}_{z}$ peak amplitude at the level of the heterogeneity (the second peak on Fig. 7 7 ) depends on the $\sigma_{\max }$ value of the heterogeneity, on its width and on its distance to the tip. It was found that a width of Ly/6 (a bloc composed of one twin and one $\mathrm{E}$ unit) and a $\sigma_{\max }$ of $10 \mathrm{GPa}$, constitute a region that can never be fractures whatever the distance to the tip, i.e. whatever the stage of development of the $\mathrm{PZ}$ when it reaches the obstacle. For thinner heterogeneities, $\Delta \tilde{u}_{z}$ does not systematically cross the threshold. It means that if a brittle PZ is initiated far enough from a heterogeneity, it can pass through without triggering plasticity. This is, in essence, what is observed in the atomistic simulations.

Starting from these generic results, $\sigma_{\max }$ profiles can be built which reproduce the crack behaviors corresponding to specific cavity configurations. The main behaviors are summarized below and translated in cohesive heterogeneities. Incipient dislocations are frequently formed when a twin structural unit, or an E unit with a small cavity, is absorbed into the PZ. The typical length scales for heterogeneities should therefore be multiples of the size of such structural units (Ly/12). It is also observed that cavity (3), in configurations (c) and (d), is an obstacle (Fig. 6 $\mathrm{d}$ ). It is therefore represented by a cohesive bloc of size Ly/6 and high stress (10 GPa). When sampling the behavior of configuration (e) (appendix), it was found that dislocations can be emitted right at the tip, i.e. there are positions along the GB where the brittle PZ initiation criterion is not satisfied. This is the case at almost every inter-cavity spacing (positions 6e in Fig. 10p and $4 \mathrm{e}, 2 \mathrm{e}, 8 \mathrm{e}$ in Fig. 10k) and therefore, regions of $\sigma_{\max }$ beyond 5.5 GPa exists at these positions. They appear as peaks on the profile on Fig. 8 $\mathrm{b}$. In between these peaks, brittle $\mathrm{PZ}$ initiation is possible, and therefore the cohesive stress should be lower or close to $5.5 \mathrm{GPa}$. The region between cavity 3 and 5 has received a special attention because atomistic data showed a change of slope in the crack opening profile in this region. The $\sigma_{\max }$ profile proposed captures these features [36]. In addition to these qualitative constraints, the $\sigma_{\max }$ profile should respect the average value given in Tab. [II] The amplitude and thickness of the heterogeneities were adjusted to respect this constraint. A similar reasoning was applied to configuration (d). The two profiles are given on Fig. 8 ,

The heterogeneous CZ models were tested by placing a crack tip at different positions along the $\sigma_{\max }$ profile and calculating the PZ equilibrium for a range of loads. The model reproduces typical behaviors found in the atomistic simulations: (i) dislocation emission at the crack tip $\left(\Delta \tilde{u}_{z}(y)\right.$ curves labeled (1) on Fig. $8 \mathrm{~b}$ and b), when it is located in a region of high cohesion $\left(\Delta \tilde{u}_{z}(y)\right.$ becomes larger the threshold at $0.04 \mathrm{~nm}$ ); (ii) brittle $\mathrm{PZ}$ initiation, followed by dislocation emission ahead of the tip (curves (2) and (3) on Fig. 8 a ) when the crack tip is in a low cohesion zone but in the vicinity of high $\sigma_{\max }$ region. (iii) initiation and propagation (curves (2) and (3) on Fig. 8b) when the PZ goes through thin regions of high $\sigma_{\max }$.

Finally, in this section it was shown that the apparent complexity of the atomistic results derives from simple characteristics of cohesion heterogeneities: brittle PZ initiation requires a drop of $\sigma_{\max }$ below $5.5 \mathrm{GPa}$ and, after initiation, brittle propagation occurs if no high cohesion zones thicker than Ly/6 $(1.15 \mathrm{~nm})$ exists. The comparison between the $\sigma_{\max }$ profiles of configurations (d) (ductile) and (e) (brittle) (Fig. 8 a vs. b), shows that the origin of the transition to brittleness is a drop of the thickness of the high cohesion zones. The heterogeneous cohesive zone model is generic and could apply to forms of embrittlement other than nanoscale cavities. 


\section{DISCUSSION AND CONCLUSION}

The heterogeneous cohesive zone model has shown that two processes are involved in the formation of a brittle crack. The initiation of a brittle fracture process zone (PZ) is the first step. It requires a very significant drop in cohesive stress $(5.5 \mathrm{GPa}$ for $\mathrm{Al}$, which is half of the theoretical fracture stress given by the rigid separation calculations in Tab. III). Once the formation of the brittle PZ is initiated, it can grow and lead to brittle propagation, only if no high cohesion zones are met. In an attempt to provide a quantitative link between the characteristics of the cavities (density and size) and these two processes (initiation or arrest) we first have to describe how initiation occurs in the simulations, in the light of the cohesive zone model, to define the appropriate area over which the critical density of cavities for initiation is defined.

A sampling composed of 70 crack configurations was analyzed. They were gathered from the simulations carried on cavity configurations (a) to (e), with 10 different crack tip positions each, and on the $\{554\}$ single crystal with 20 tip positions, since both right and left tips can be used in this case. No initiation occurred in configuration (a). Only one initiation was seen in configuration (b), while multiple ones were obtained in (c), (d) and (e). Initiation requires "large" cavities. Their characteristics are defined by the triplet: cavity surface coverage $f_{S}$ (the projected area in the GB plane, divided by the area of a band of structural units D or E running all along the tilt axis, i.e. a thin band of dimension $\mathrm{Ly} / 12 \times \mathrm{Lx})$, volume of the cavity (in $\mathrm{a}_{0}^{3}$ ) and aspect ratio (cavities are elongated in the direction of the tilt axis). In our case, the cavities have an approximate width of Ly/12, so $f_{S}$ is also the length of the cavity and the inter-cavity spacing along $\mathrm{x}$ (the tilt axis) is $\operatorname{Lx}\left(1-f_{S}\right)$. The smallest cavities which lead to PZ initiation are: cavity 4 of (b) $(0.28 / 1.01 / 1.69)$, cavity 3 of (e) $(0.29 / 1.39 / 1.72)$ and cavity 5 of $(\{554\}(\mathrm{e}))(0.49 / 5.55 / 2.90)$. The largest cavities which did not lead to $\mathrm{PZ}$ initiation are: cavity 4 of (a) $(0.35 / 3.81 / 2.01)$, cavity 5 of (b) $(0.25 / 0.85 / 1.48)$, cavity 6 of (e) $(0.23 / 1.64 / 1.36)$ and cavity 2 of $(\{554\}(\mathrm{e}))$ $(0.25 / 1.74 / 1.48)$. These bounds suggest there exist a critical cavity for initiation, in the GB case, with characteristics $(0.30 / 1.4 / 1.77)$. This corresponds to a length of $1 \mathrm{~nm}$ and an inter-cavity spacing of $2.4 \mathrm{~nm}$ (along $\mathrm{x}$ ). Note that, in this size/density range, the response of the system is essentially 2D: the crack opens or an infinite dislocation pops out of the crack tip. The cavities interact, at this high density. For example, the largest cavity of configuration (a) $(0.35 / 3.81 / 2.01)$ is beyond the critical size, but is surrounded by small cavities and does not lead to PZ initiation. Furthermore, in defining the local density of cavities, we remind that if the crack tip is constructed at the cavity, the latter is immediately absorbed and plays a very limited role in the formation of the PZ. Therefore, the cavity should be at a certain distance from the tip, under the influence of the stress concentration.
The virial traction stress profile in the single crystal case (without cavities) loaded slightly belong $\mathrm{k}_{I e}$ gives a stress of $5 \mathrm{GPa}$ at a distance $2 \mathrm{a}_{0}$ from the tip. A stress high enough to start having an effect on a cavity with a size larger than the critical one. This length is of the order of the size of a bloc composed of one E and one D structural unit $\left(\sim 2.8 \mathrm{a}_{0}\right)$. So the area over which we define the local GB coverage by cavities, for crack initiation, is $\mathrm{Ly} / 6$ $\times$ Lx. It leads to an upper bound for the critical coverage of $f_{S} / 2=15 \%$ (half the $f_{S}$ of the critical cavity) which compares well with the average GB coverage of the brittle configuration (e): $14 \%$. At constant cavity size and decreasing the coverage down to $7.5 \%$ by increasing the box thickness from Lx to $2 \mathrm{Lx}$, configuration (f) shows no sign of embrittlement $\left(\mathrm{k}_{I e}\right.$ is the same as without cavities (Fig. 5] and 100). A new phenomenon appears: the formation of half loops in between the cavities.

One can wonder at which cavity size and inter-cavity spacing do half loops appear. Some preliminary calculations were made at intermediate box thicknesses $1.5 \mathrm{Lx}$ and 1.8 Lx. Five configurations (numbered 0, 1, 3, 4 and 5) of configuration (e) (Fig. 10 c) were selected [36]. Upon mechanical loading, initiation was observed only once and also occurs at a local coverage of $15 \%$ (at 1.5 Lx). A local coverage of $11 \%$ led to immediate dislocation emission at the tip. One artificial cavity configuration was constructed by selecting the largest cavity and placing it in a chessboard arrangement 36] (the cavity size is about $2 \mathrm{~nm}$, the inter-cavity spacing in the $\mathrm{x}$ direction is about $4 \mathrm{~nm}$ and box thickness $1.8 \mathrm{Lx}$ ). Half loops are emitted in this case. Therefore the simulations show that not only the coverage should be high $(15 \%$ or beyond) but also the cavities should be small (1.8 nm led to initiation and $2.2 \mathrm{~nm}$ led to dislocation emission) and the inter-cavity spacing should also be small (below $4 \mathrm{~nm}$ ). A complete understanding of half loop emission and the corresponding size effects should consider the shielding of the shear stress produced by an individual cavity, the role of line tension [47] and stress concentration at the tip. It is left for future work.

Once the formation of the brittle $\mathrm{PZ}$ is initiated, it can grow and eventually reach propagation, but only if no hard cohesion zones are met. Out of the 50 GB configurations, 28 showed initiation which lead to 4 true propagation, all for configuration (e) (out of 10). A number of cavities were identified as "obstacles", i.e. that they did not decrease cohesion enough, locally, to let the brittle crack pass. The corresponding average local coverage, rejecting the small cavities i.e. cavities from configurations (a) and (b), is $9.2 \%$ (8 obstacles identified, of average characteristics $(0.18 / 1.06 / 1.09))$. This corresponds to a region with a small cavity $(0.6 \mathrm{~nm}$ and inter-cavity spacing of $2.8 \mathrm{~nm}$ ). On a thicker system (thickness $1.5 \mathrm{Lx}$ ), the brittle PZ stops when a half loop is emitted in the vicinity of cavity 5 which size is $1.4 \mathrm{~nm}$ and inter-cavity spacing $3.6 \mathrm{~nm}$ (local coverage 11\%) 36].

The $\{554\}$ single crystal behaves in a similar way to the $\mathrm{GB}$ : the $\mathrm{k}_{I e}$ values are spread, $\mathrm{PZ}$ initiation and crack 
arrest are observed. The conclusions are therefore not specific of the E structural unit. Nevertheless, the single crystal is much tougher than the GB as shown by the values of the average cohesive stress (6.77 GPa vs. 6.00 for the GB in Tab. III) and the work of separation $\left(3.59 \mathrm{~J} / \mathrm{m}^{2}\right.$ vs. $\left.3.27 \mathrm{~J} / \mathrm{m}^{2}\right)$. The cavity size and density for embrittlement will be significantly higher, of the order of $(0.49 / 5.55 / 2.90)$ (local density $25 \%)$ or higher. This means that the $\mathrm{E}$ unit plays a role in the fracture mechanism. Activation barrier energy calculations (not shown) suggest self interstitial injection is favored in the direction of the core of the E unit.

A recent experimental study, on another face centered cubic system, reports a critical coverage of GBs by nanoscale bubbles of size $1.5 \mathrm{~nm}$ and spacing $4 \mathrm{~nm}$ [49]. If the spacing is multiplied by 2 , fracture becomes intergranular ductile. These observations are in good agreement with our simulations (owing to the simplifications of the model, especially the absence of any thermally activated events and the choice of a crystallographic orientation which favors ductility): brittle crack initiation was obtained with the cavities of configuration (e) (tested with box thicknesses Lx, 1.5 Lx and 1.8 Lx) which sizes are in between 1 and $2.2 \mathrm{~nm}$ and inter-cavity spacing in the $\mathrm{x}$ direction is in between 1.5 and $3.3 \mathrm{~nm}$, while configuration (f), obtained by multiplying the inter-cavity distance, in $\mathrm{x}$, by a factor 2 , is ductile.

In conclusion, atomistic simulations have shown that a dense distribution of nanoscale bubbles could render a ductile GB brittle. By "brittle", it is meant that crack propagation occurs along the GB core at the expense of massive dislocation emission and crack tip blunting, but with, eventually, the contribution of nanoscale plastic events. It is worth recalling that the crystallographic orientation chosen in this study favors a ductile response of the tip, and therefore the critical damage found is an upper-bound. The heterogeneous cohesive zone model has shown that the cohesive stress must drop, locally, by more than $50 \%$ to enable fracture initiation. Comparatively, the corresponding coverage by cavities is modest, of the order of $15 \%$. A schematic representation of the arrangement of the cavities at this critical coverage is given in [36]. Note that small cavity sizes (in between $1 \mathrm{~nm}$ and $2 \mathrm{~nm}$ ) and inter-cavity spacings (below $4 \mathrm{~nm}$ ) play a crucial role, otherwise dislocation half loops can be easily emitted, in this orientation. The interpretation of the cohesion drop is not a simple section reduction leading to the cleavage of the inter-cavity spacings. The fracture mechanism, as far as energy minimization simulations can tell, is a combination of an injection of self-interstitials from the side of the cavities and, eventually, localized plastic events (stacking fault tetrahedra in the tough regions, especially in the single crystal case, and local glide in between the cavities). The corresponding fracture energy is two to three times higher than the ideal cleavage one. The comparison between the GB case and the single crystal shows that the E units does not affect dislocation emission, but do contribute significantly (a)

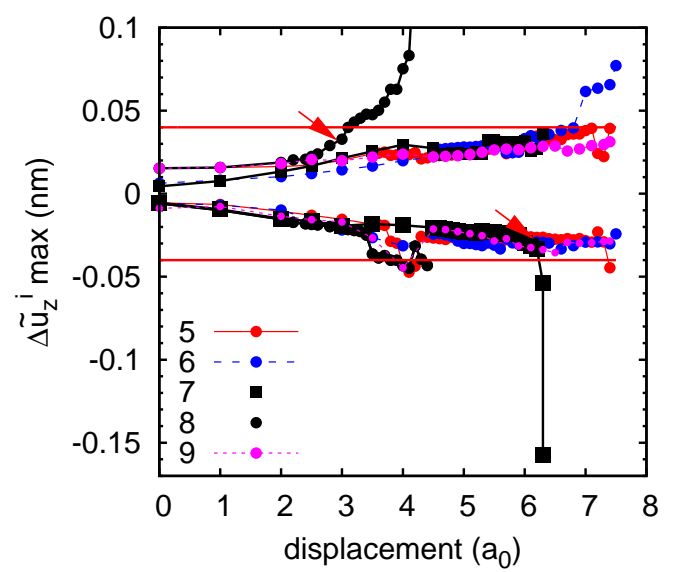

(b)

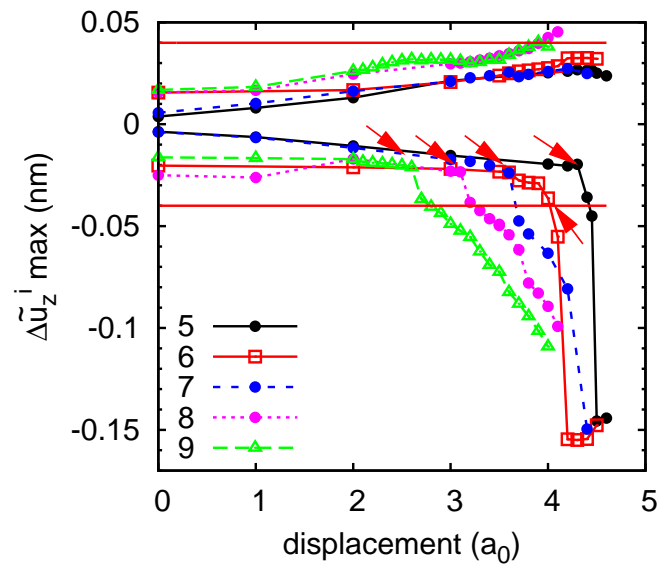

Figure 9. (Color online) Variation of the maximum displacement shift with the applied mechanical load (displacement on the border) for (a) cavity configuration (e) and (b) a configuration without cavities, for different initial crack tip positions. The threshold for dislocation emission $0.04 \mathrm{~nm}$ is represented by red lines. Positive and negative values refer to profiles above and below the crack plane respectively. The calculations are done on the large system.

to the cavity growth (without E units, growth occurs at higher loads and requires larger cavities and higher coverages for initiation). Dividing the coverage by a factor 2 (configuration (f)), for example because of aging of the bubble population, would make barely no difference with the undamaged GB because half loops are emitted in between the cavities. Once initiated, propagation can be stopped if regions of high local cohesive stress are met. The generic heterogeneous cohesive zone model gives the characteristics of such obstacle. In particular, its width is important (10 GPa over $1.15 \mathrm{~nm}$ ). This is particularly relevant in the case where the cavities are small and dense and the response in essentially 2D. The simulations show that cavities of size $0.6 \mathrm{~nm}$ with an inter-cavity spacing $2.8 \mathrm{~nm}$ are efficient obstacles. In the case where the cavities are larger and the inter-cavity spacing too, half loop emission is the key element. First results show that an inter-cavity spacing beyond $4 \mathrm{~nm}$ would act as an effi- 
cient obstacle to brittle crack propagation by enabling half loop formation.

This points to the dual aspect of the study: the ways to prevent or decrease the sensitivity to embrittlement. The high cavity coverage necessary for brittle propagation emphasizes the ease by which dislocation half loops can appear at crack tips. This suggests that a distribution, even at low density, of soft, coherent, precipitates of nano scale length (as small as $4 \mathrm{~nm}$ ) could act as sources of dislocation loops and could significantly enhance the resistance of the GBs to embrittlement.

\section{ACKNOWLEDGMENTS}

This work received a financial support from the French Agence Nationale de la Recherche (ANR), under grant Blanc10-19424. It is a pleasure to thank N. Combe (professor at CEMES, Toulouse) for his comments during the preparation of the manuscript.

\section{Appendix A: Additional data concerning dislocation emission and brittle cracking}

The critical slip distribution, just before the dislocation is emitted, as modeled by Rice [45], starts at the crack tip with a value which is the maximum of the distribution. It corresponds to the unstable staking $(0.08$ $\mathrm{nm}$ in our case). The distribution extends ahead of the tip (the slip decreases to zero). Doing so, some atoms, close to the tip, experience a low shear stress because they are sheared beyond the maximum resistance shear stress (Fig. 6 b), i.e. beyond $0.04 \mathrm{~nm}$. Others, further away, are sheared below this value. This is not what the data show (Fig. 9). Emission can occur with a maximum slip anywhere in between 0.02 and $0.04 \mathrm{~nm}$ (between 1/4 and $1 / 2$ of the Rice model). Note that the truly brittle configuration (e), with tip positions 5, 6 and 9 (Fig. 9a), never exceeds $0.04 \mathrm{~nm}$, except for large loads where some stacking fault tetrahedra appear during crack propagation and perturb the measure of the shear localization. The limitation in Rice's analysis is that he assumes every atom follows the same Peierls potential, calculated in the bulk, while it is clear that an atom at the crack surface in not bound like an atom in the bulk. The same holds for the atoms in the process zone, in the GB core, where dislocation emission occurs. While the Rice model is very good in mode II [38], when the crack tip is sharp and its shape is preserved during emission, it requires modifications when emission is at an angle and steps are formed [50, 51].
[1] M. Tanaka, E. Tarleton, and S. G. Roberts, Acta Materialia 56, 5123 (2008).

[2] S. J. Zhou, A. E. Carlsson, and R. Thomson, Phys. Rev. B 47, 7710 (1993).

[3] S. J. Zhou, A. E. Carlsson, and R. Thomson, Phys. Rev. Lett. 72, 852 (1994).

[4] R. J. Zamora, A. K. Nair, R. G. Hennig, and D. H. Warner, Phys. Rev. B 86, 060101(R) (2012).

[5] Y. Sun, Q. Peng, and G. Lu, Phys. Rev. B 88, 104109 (2013).

[6] J. Song and W. A. Curtin, Nature Materials 12, 145 (2013).

[7] P. A. Gordon and T. Neeraj, acta materialia 57, 3091 (2009).

[8] W. T. Geng, A. J. Freeman, and G. B. Olson, Phys. Rev. B 63, 165415 (2001).

[9] R. Janish and C. Elsässer, Int. J. Mat. Res. 100, 1488 (2009).

[10] G. Duscher, M. F. Chisholm, U. Alber, and M. Rühle, Nature Materials 3, 621 (2004).

[11] A. Y. Lozovoi, A. T. Paxton, and M. W. Finnis, Phys. Rev. B 74, 155416 (2006).

[12] J. Kang, G. C. Glatzmaier, and S.-H. Wei, Phys. Rev. Lett. 111, 055502 (2013).

[13] J. R. Rice and J. Wang, Materials Science and Engineering A 107, 23 (1989).
[14] A. Van der Ven and G. Ceder, Phys. Rev. B 67, 060101(R) (2003).

[15] R. Kirchheim, B. Somerday, and P. Sofronis, acta mater. 99, 87 (2015).

[16] Y. Fukai and N. Ōkuma, Phys. Rev. Lett. 73, 1640 (1994).

[17] M. Nagumo, Materials Science and Technology 20, 940 (2004).

[18] H. Momida, Y. Asari, Y. Nakamura, Y. Tateyama, and T. Ohno, Phys. Rev. B 88, 144107 (2013).

[19] S. Li, Y. Li, Y.-C. Lo, T. Neeraj, R. Srinivasan, X. Ding, J. Sun, L. Qi, P. Gumbsch, and J. Li, International Journal of Plasticity 74, 175 (2015).

[20] A. Tehranchi, X. Zhang, G. Lu, and W. A. Curtin, Modelling Simul. Mater. Sci. Eng. 25, 025001 (2017).

[21] Y. Wang, D. Connétable, and D. Tanguy, Phys. Rev. B 91, 094106 (2015).

[22] D. Xie, S. Li, M. Li, Z. Wang, P. Gumbsch, J. Sun, E. Ma, J. Li, and Z. Shan, Nature Communications 7, 13341 (2016).

[23] D. Tanguy, D. Delafosse, and M. Razafindrazaka, Phil. Mag. 90, 1415 (2010).

[24] J. D. Rittner and D. N. Seidman, Phys. Rev. B 54, 6999 (1996).

[25] J.-P. Du, Y.-J. Wang, Y.-C. Lo, L. Wan, and S. Ogata, Phys. Rev. B 94, 104110 (2016). 


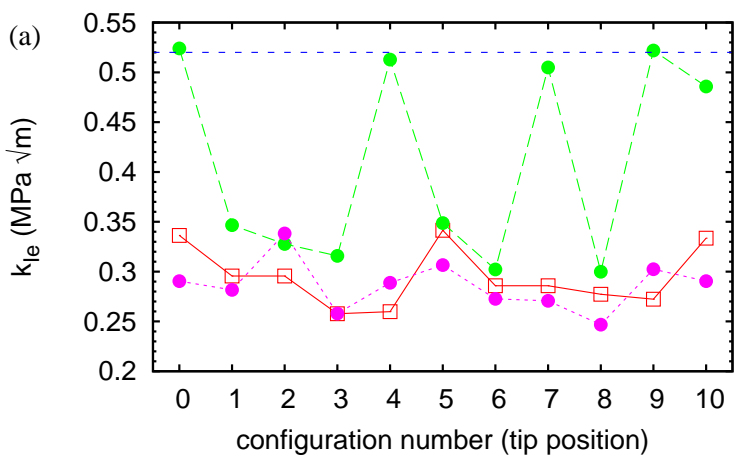

(b)
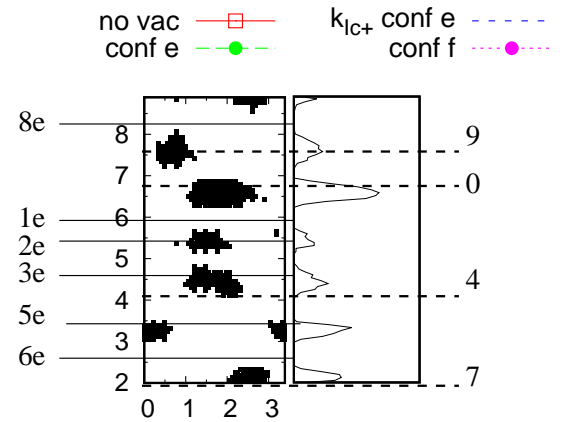

(c)

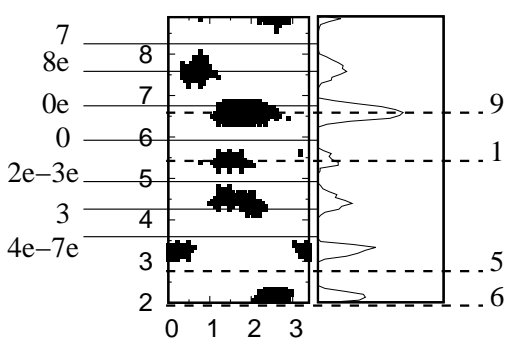

Figure 10. (Color online) (a) Comparison of the critical stress intensity factor for dislocation emission without cavities, in the case of the configuration (e) and (f) (small systems). Note the good agreement between the $\mathrm{k}$ values obtained by the fitting procedure and the one obtained from $W_{\text {sep }}\left(k_{I c}^{+}\right)$in case of crack propagation. (b, c) The cavities of configuration (e) are viewed in the crack plane $(\mathrm{x}, \mathrm{y})$, and the corresponding porosity profile are given. The horizontal lines represent the different positions where the crack tip is initially situated. On the left are the labels which correspond to simulations where dislocation emission occurred. When a label appears twice, like 7 and 7 e, it means that a brittle process zone was initiated and that dislocation emission occurred ahead of the crack tip. The second line, labeled with the extension "e", marks the position where the dislocation was emitted. On the right, with dashed lines, are the tip positions which lead to brittle crack propagation. (c) is the same as (b) but for the large system with a large crack.
[26] D. N. Pawaskar, R. Miller, and R. Phillips, Phys. Rev. B 63, 214105 (Structure and energetics of long-period tilt grain boundaries using an effective Hamiltonian).

[27] Y. Mishin, D. Farkas, M. J. Mehl, and D. A. Papaconstantopoulos, Phys. Rev. B 59, 3393 (1999).

[28] E. Vamvakopoulos and D. Tanguy, Phys. Rev. B 79, 094116 (2009).

[29] K. Carling, G. Wahnström, T. R. Mattsson, A. E. Mattsson, N. Sandberg, and G. Grimvall, Phys. Rev. Lett. 85, 3862 (2000).

[30] A. Glensk, B. Grabowski, T. Hickel, and J. Neugebauer, Phys. Rev. X 4, 011018 (2014).

[31] L. Christodoulou and H. M. Flower, Acta Metallurgica 28, 481 (1980).

[32] H. Wang, D. Rodney, D. Xu, R. Yang, and P. Veyssière, Phys. Rev. B 84, 220103(R) (2011).

[33] F. Cleri, S. R. Phillpot, D. Wolf, and S. Yip, J. Am. Ceram. Soc. 81, 501 (1998).

[34] D. Tanguy, Phys. Rev. B 76, 144115 (2007).

[35] J. Weertman, Dislocation Based Fracture Mechanics, reprint edition (World Scientific Publishing, Singapore, 1998).

[36] See Supplemental Material at [URL will be inserted by publisher] for crack size effects, simulation of crack opening profiles, critical cavity coverages and a movie of crack propagation.

[37] D. Tanguy, M. Razafindrazaka, and D. Delafosse, acta mater. 56, 2441 (2008).

[38] K. Gouriet and D. Tanguy, Phil. Mag. 92, 1663 (2012).

[39] F. Cleri, S. R. Phillpot, and D. Wolf, Interf. Sci. 7, 45 (1999).

[40] V. Yamakov, E. Saether, D. Phillips, and E. Glaessgen, J. Mech. Phys. Solids 54, 1899 (2006).

[41] P. Gumbsch, S. J. Zhou, and B. L. Holian, Phys. Rev. B 55, 3445 (1997).

[42] R. Janisch, N. Ahmed, and A. Hartmaier, Phys. Rev. B 81, 184108 (2010).

[43] J. Song, W. A. Curtin, T. K. Bhandakkar, and H. J. Gao, acta mater. 58, 5933 (2010).

[44] F. Erdogan, G. D. Gupta, and T. S. Cook, "Mechanics of fracture, methods of analysis and solutions of crack problems," (Springer-Science, 1973) Chap. 7, p. 368.

[45] J. R. Rice, J. Mech. Phys. Solids 40, 239 (1992).

[46] J. R. Rice and R. Thomson, Phil. Mag. 29, 73 (1974).

[47] G. Monnet, acta mater. 55, 5081 (2007).

[48] E. Bitzek, J. R. Kermode, and P. Gumbsch, Int. J. Fract. 191, 13 (2015).

[49] T. Miura, K. Fujii, and K. Fukuya, Journal of nuclear materials 457, 279 (2015).

[50] Y.-M. Juan, Y. Sun, and E. Kaxiras, Phil. Mag. Lett. 73, 233 (1996).

[51] P. Andric and W. A. Curtin, J. Mech. Phys. Solids 106, 315 (2017). 